\title{
Polar coding for interference networks
}

\author{
Lele Wang and Eren Şaşoğlu
}

\begin{abstract}
A polar coding scheme for interference networks is introduced. The scheme combines Arlkan's monotone chain rules for multiple-access channels and a method by Hassani and Urbanke to 'align' two incompatible polarization processes. It achieves the Han-Kobayashi inner bound for two-user interference channels and generalizes to interference networks.
\end{abstract}

\section{INTRODUCTION}

Interference is one of the fundamental challenges in wireless communication. When multiple sender-receiver pairs communicate simultaneously over a shared medium, the signal arrived at each receiver is a mixture of its intended signal and undesired signals from all other transmitters. Therefore, even in the absence of noise, transmission from a sender to its receiver is limited by the presence of transmission from other parties.

The two-user memoryless interference channel models the simplest such communication setting. It is described by channel input alphabets $\mathcal{X}, \mathcal{W}$, output alphabets $\mathcal{Y}, \mathcal{Z}$, and for all $(x, w, y, z) \in \mathcal{X} \times \mathcal{W} \times \mathcal{Y} \times \mathcal{Z}$, the probability $P(y, z \mid x, w)$ of receiving $(y, z)$ when $(x, w)$ are input to the channel (Figure 1).

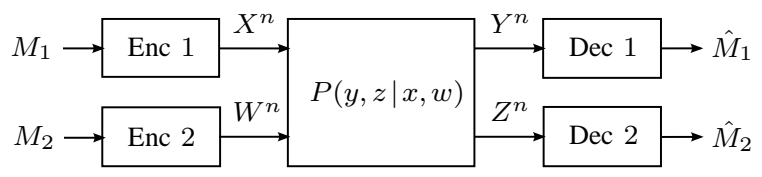

Fig. 1. Two-user interference channels.

A $\left(2^{N R_{1}}, 2^{N R_{2}}, N\right)$ code for the two-user interference channel consists of

- two encoding functions $x^{N}\left(M_{1}\right)$ and $w^{N}\left(M_{2}\right)$, defined for messages $M_{1} \in\left[1: 2^{N R_{1}}\right]$ and $M_{2} \in\left[1: 2^{N R_{2}}\right]$.

- two decoding functions $\hat{m}_{1}\left(y^{N}\right)$ and $\hat{m}_{2}\left(z^{N}\right)$, defined for each received sequence $y^{N} \in \mathcal{Y}^{N}$ and $z^{N} \in \mathcal{Z}^{N}$.

Messages $M_{1}$ and $M_{2}$ are assumed to be uniformly distributed. The average probability of error is defined as $P_{e}^{(N)}=$ $\mathrm{P}\left\{\left(\hat{M}_{1}, \hat{M}_{2}\right) \neq\left(M_{1}, M_{2}\right)\right\}$. A rate pair $\left(R_{1}, R_{2}\right)$ is achievable if there exists a sequence of $\left(2^{N R_{1}}, 2^{N R_{2}}, N\right)$ codes with $\lim _{N \rightarrow \infty} P_{e}^{(N)}=0$. The capacity region is the closure of the set of achievable rate pairs.

The capacity region of the two-user interference channel is not known in general. The best known inner bound to the capacity region was given by Han and Kobayashi in [1]. Our

L. Wang is with the Department of Electrical and Computer Engineering, University of California, San Diego, La Jolla, CA 92093 USA (email: lew001@ucsd.edu).

E. Şaşoğlu is with the Department of Electrical Engineering and Computer Sciences, University of California, Berkeley, CA 94720 USA (email: eren@eecs.berkeley.edu). aim here is to show the achievability of this inner bound by polar coding techniques.

The Han-Kobayashi scheme consists in splitting each sender's message into two parts, and letting each receiver decode one part of the interfering sender's message in addition to both parts of its own sender's message. This creates a threesender multiple-access channel (MAC) for each receiver, and the code rates are required to satisfy both MACs simultaneously. Since these MACs share two of their three senders, the situation is similar to a compound setting, in which codes must be designed to perform well simultaneously over several (in this case, two) MACs.

Given these observations, one may hope to apply the standard results on MACs to design polar codes for the interference channel. In particular, the corner points of a MAC's capacity region are known to be achievable by standard polar coding techniques [2]. This readily implies the achievability of the entire MAC capacity region by polar coding, since all achievable points can be turned into corner points by the rate-splitting techniques of [3]. Unfortunately, rate-splitting techniques do not generalize in a straightforward manner to the compound setting. In particular, it is shown in a parallel study [4] that standard applications of rate-splitting techniques fail to achieve optimal compound rates in general. This makes it unclear whether polar coding techniques can be combined with ratesplitting ideas to achieve the Han-Kobayashi inner bound.

Here, we show an alternative polar coding method that achieves the capacity region of compound MACs and by extension the Han-Kobayashi inner bound. The method is based on appropriately combining two techniques developed recently by Arikan [5], and Hassani and Urbanke [6]. We briefly review these techniques first.

\section{PREliminaries}

\section{A. Aligning polarized indices $([6])$}

Consider two binary-input memoryless channels $P: X \rightarrow$ $Y$ and $Q: X \rightarrow Z$ with equal symmetric capacities $I(P)=I(Q)$. Suppose we wish to design a polar code that performs well over both of these channels. For $N=2^{n}$, define $U^{N}=X^{N} G_{N}$, where $G_{N}=\left[\begin{array}{l}1,0 \\ 1,1\end{array}\right]^{\otimes n} B_{N}$ is the standard polar transformation. Here, $\otimes n$ denotes the $n$th Kronecker power and $B_{N}$ is the 'bit-reversal' permutation. Define the channels $P_{i}: U_{i} \rightarrow Y^{N} U^{i-1}$ and $Q_{i}: U_{i} \rightarrow Z^{N} V^{i-1}$ and sets

$$
\begin{aligned}
& \mathcal{G}_{Y}=\left\{i \in[1: N]: I\left(P_{i}\right)>1-2^{-N^{\beta}}\right\}, \\
& \mathcal{G}_{Z}=\left\{i \in[1: N]: I\left(Q_{i}\right)>1-2^{-N^{\beta}}\right\}, \\
& \mathcal{B}_{Y}=\left\{i \in[1: N]: I\left(P_{i}\right)<2^{-N^{\beta}}\right\}, \\
& \mathcal{B}_{Z}=\left\{i \in[1: N]: I\left(Q_{i}\right)<2^{-N^{\beta}}\right\} .
\end{aligned}
$$


for some $\beta<1 / 2$. Standard polarization results imply that $\left|\mathcal{G}_{Y}\right| / N \approx I(P)=I(Q) \approx\left|\mathcal{G}_{Z}\right| / N$ for large $N$, and thus almost all bit indices belong to one of the following four sets:

$$
\begin{aligned}
\mathcal{A}_{\mathrm{I}} & =\mathcal{G}_{Y} \cap \mathcal{G}_{Z}, \\
\mathcal{A}_{\mathrm{II}} & =\mathcal{G}_{Y} \cap \mathcal{B}_{Z}, \\
\mathcal{A}_{\mathrm{III}} & =\mathcal{B}_{Y} \cap \mathcal{G}_{Z}, \\
\mathcal{A}_{\mathrm{IV}} & =\mathcal{B}_{Y} \cap \mathcal{B}_{Z} .
\end{aligned}
$$

It suffices to discuss only the bit indices of the above four types, and assume that the remaining bit values are fixed and revealed to all receivers. Note that type-I indices see clean channels for both $P$ and $Q$ and thus can carry information. Similarly, type-IV indices are bad for both channels and can be fixed. Type-II and III indices are incompatible, i.e., they are good for one channel and bad for the other. Moreover, the fraction $\left(\left|\mathcal{A}_{\text {II }}\right|+\left|\mathcal{A}_{\text {III }}\right|\right) / N$ of incompatible indices is nonnegligible in general [7], and therefore standard polar coding does not achieve the compound capacity of arbitrary channels $P$ and $Q$.

Hassani and Urbanke propose a simple solution to this problem, which aligns the good indices of the two channels. Given two independent binary-input memoryless channels $P: X_{1} \rightarrow Y_{1}$ and $Q: X_{2} \rightarrow Y_{2}$, define the binary-input channels

$$
\begin{aligned}
& (P, Q)^{-}\left(y_{1}, y_{2} \mid u_{1}\right)=\sum_{u_{2}} \frac{1}{2} P\left(y_{1} \mid u_{1} \oplus u_{2}\right) Q\left(y_{2} \mid u_{2}\right), \\
& (P, Q)^{+}\left(y_{1}, y_{2}, u_{1} \mid u_{2}\right)=\frac{1}{2} P\left(y_{1} \mid u_{1} \oplus u_{2}\right) Q\left(y_{2} \mid u_{2}\right),
\end{aligned}
$$

and note that

$$
\begin{aligned}
& I\left((P, Q)^{-}\right) \leq \min \{I(P), I(Q)\} \\
& I\left((P, Q)^{+}\right) \geq \max \{I(P), I(Q)\} .
\end{aligned}
$$

Now let $i$ and $j$ be a type-II and a type-III index, respectively. That is,

$$
\begin{aligned}
& I\left(P_{i}\right) \approx 1 \text { and } I\left(P_{j}\right) \approx 0, \\
& I\left(Q_{i}\right) \approx 0 \text { and } I\left(Q_{j}\right) \approx 1 .
\end{aligned}
$$

It then follows from (2) that

$$
\begin{gathered}
I\left(\left(P_{i}, P_{j}\right)^{-}\right) \approx 0 \quad \text { and } I\left(\left(P_{i}, P_{j}\right)^{+}\right) \approx 1 \\
I\left(\left(Q_{i}, Q_{j}\right)^{-}\right) \approx 0 \quad \text { and } I\left(\left(Q_{i}, Q_{j}\right)^{+}\right) \approx 1,
\end{gathered}
$$

In words, combining two incompatible indices results in an almost perfect 'plus' channel and almost useless 'minus' channel, regardless of the underlying channel. This 'aligns' the mutual informations for such indices. Taking two blocks of $U^{N}$, one can combine almost all type-II indices from one block with type-III indices from the other block, since $\left|\mathcal{A}_{\text {II }}\right| / N \approx$ $\left|\mathcal{A}_{\text {III }}\right| / N$. More precisely, suppose $\mathcal{A}_{\text {II }}=\left\{c_{1}, c_{2}, \ldots, c_{m}\right\}$ and $\mathcal{A}_{\text {III }}=\left\{d_{1}, d_{2}, \ldots, d_{n}\right\}$, where the elements are written in increasing order. Define $U^{N}=X^{N} G_{N}$ and $E^{N}=X_{N+1}^{2 N} G_{N}$. Then, combining $U_{c_{j}}$ with $E_{d_{j}}, j=1, \ldots, q=\min \{m, n\}$, and leaving the remaining symbols uncombined yields the length- $2 N$ sequence

$$
\begin{aligned}
\tilde{U}^{2 N}= & \left(U^{c_{1}-1}, E^{d_{1}-1}, U_{c_{1}} \oplus E_{d_{1}}, E_{d_{1}}\right. \\
& \cdots \\
& U_{c_{q-1}+1}^{c_{q}-1}, E_{d_{q-1}+1}^{d_{q}-1}, U_{c_{q}} \oplus E_{d_{q}}, E_{d_{q}}, \\
& \left.U_{c_{q}+1}^{N}, E_{d_{q}+1}^{N}\right) .
\end{aligned}
$$

Then, the mutual informations of channels $\tilde{U}_{i} \rightarrow Y^{2 N} \tilde{U}^{i-1}$ and $\tilde{U}_{i} \rightarrow Z^{2 N} \tilde{U}^{i-1}$ are aligned for the combined indices $\tilde{U}_{i}=U_{c_{j}} \oplus E_{d_{j}}$ and $\tilde{U}_{i}=E_{d_{j}}$, and unchanged for the remaining ones. Note again that the indices in $\mathcal{A}_{\text {III }}$ of the first block and $\mathcal{A}_{\text {II }}$ of the second block are not combined with each other and remain incompatible. This ensures that the combined indices are polarized as desired. The fraction of incompatible indices is thus halved by this alignment, to $\left(\left|\mathcal{A}_{\mathrm{II}}\right|+\left|\mathcal{A}_{\mathrm{III}}\right|\right) / 2 N$. Recursively aligning the indices $k$ times in this fashion then reduces this fraction to $\left(\left|\mathcal{A}_{\text {II }}\right|+\left|\mathcal{A}_{\text {III }}\right|\right) / 2^{k} N$, and thus the rate $I(P)=I(Q)$ can be achieved on both channels by picking a large $k$.

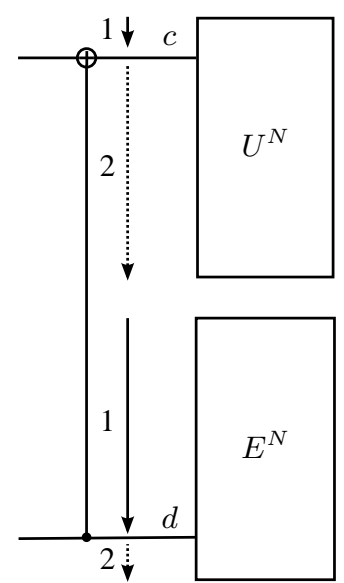

(a)

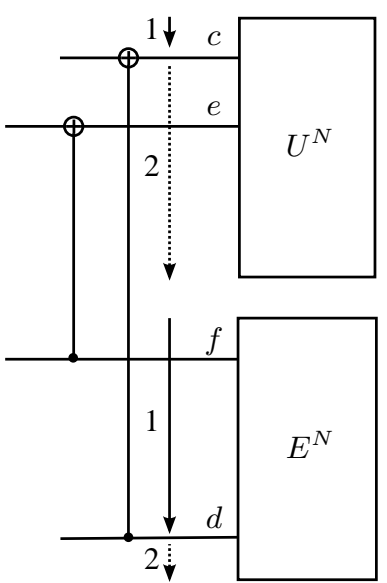

(b)
Fig. 2. Alignment of the incompatible indices

To show proper alignment of incompatible indices and its corresponding decoding order, consider an example where $\mathcal{A}_{\text {II }}=\{c\}$ and $\mathcal{A}_{\text {III }}=\{d\}$. We combine $U_{c}$ from block 1 with $E_{d}$ from block 2 as in Figure 2 (a). Decoding is thus done in the following order. Variables along the solid line arrows should be decoded before the variables along the dash line arrows. Variables along arrows with the same number can be decoded parallelly. Figure 2 (b) shows an example where improper combining violates the successive decodability. Here in order to decode $U_{c} \oplus E_{d}$, one needs to know $E^{d-1}$, and in particular $E_{f}$. However, the decoding of $E_{f}$ involves $U_{e}$, which won't be available before knowing $U_{c} \oplus E_{d}$. Therefore, it is crucial to order type II and type III indices in increasing order and combine the $j$-th type II index from one block with the $j$-th type III index from another independent block.

\section{B. 'Polar Splitting' for MAC ([5])}

Consider a two-user MAC $(\mathcal{X} \times \mathcal{W}, P(y \mid x, w), \mathcal{Y})$, where sender 1 and sender 2 wish to communicate two messages $M_{1}$ 
and $M_{2}$ to the receiver by respectively sending codewords $X^{N}\left(M_{1}\right)$ and $W^{N}\left(M_{2}\right)$ over $N$ uses of the channel. The capacity region of this channel is given by

$$
\bigcup_{p} \mathscr{R}(p)
$$

where the union is over all distributions of the form $p=$ $p(q) p(x \mid q) p(w \mid q) P(y \mid x, w)$, and $\mathscr{R}(p)$ is the set of nonnegative rate pairs $\left(R_{1}, R_{2}\right)$ satisfying

$$
\begin{aligned}
R_{1} & \leq I(X ; Y, W \mid Q), \\
R_{2} & \leq I(W ; Y, X \mid Q), \\
R_{1}+R_{2} & \leq I(X, W ; Y \mid Q) .
\end{aligned}
$$

The subset of $\mathscr{R}(p)$ satisfying $R_{1}+R_{2}=$ $I(X, W ; Y \mid Q)$ is called its dominant face, and the two points $(I(X ; Y \mid Q), I(W ; Y, X \mid Q))$ and $(I(X ; Y, W \mid Q), I(W ; Y \mid Q))$ are called its corner points. We will first consider uniform $X$ and $W$ and constant $Q$; generalizations to arbitrary distributions are discussed in Section III-B.

In [5], Arıkan develops a polar coding method that achieves the entire dominant face based on the following observations: Let $U^{N}=X^{N} G_{N}$ and $V^{N}=W^{N} G_{N}$. Consider the chain rules of the form

$$
\sum_{i=1}^{2 N} I\left(S_{i} ; Y^{N} \mid S^{i-1}\right),
$$

where $S^{2 N}=\left(S_{1}, \ldots, S_{2 N}\right)$ is a monotone permutation of $U^{N} V^{N}$, i.e., elements of both $U^{N}$ and $V^{N}$ appear in increasing order in $S^{2 N}$. Let $\mathcal{S}_{U}$ and $\mathcal{S}_{V}$ respectively denote the set of indices of $S^{2 N}$ with $S_{i}=U_{k}$ and $S_{i}=V_{k}$, and define the rates

$$
\begin{aligned}
& R_{1}=\frac{1}{N} \sum_{i \in \mathcal{S}_{U}} I\left(S_{i} ; Y^{N} \mid S^{i-1}\right), \\
& R_{2}=\frac{1}{N} \sum_{i \in \mathcal{S}_{V}} I\left(S_{i} ; Y^{N} \mid S^{i-1}\right) .
\end{aligned}
$$

The entire region $\mathscr{R}(p)$ can be achieved by polar coding if $\left(R_{1}, R_{2}\right)$ can be set to arbitrary values on the dominant face and if the mutual informations $I\left(S_{i} ; Y^{N} \mid S^{i-1}\right)$ are polarized. It turns out that these requirements are satisfied by permutations of the form $S^{2 N}=\left(U^{i}, V^{N}, U_{i+1}^{N}\right)$.

Proposition 1 ([5]). For every $\epsilon>0, \beta<1 / 2$, and rate pair $\left(I_{1}, I_{2}\right)$ on the dominant face of $\mathscr{R}(p)$, there exist an $N$ and a permutation $S^{2 N}=\left(U^{i}, V^{N}, U_{i+1}^{N}\right)$ such that

(i) $\left|R_{1}-I_{1}\right|<\epsilon$ and $\left|R_{2}-I_{2}\right|<\epsilon$,

$$
\frac{\left|\mathcal{G}^{(1)}\right|}{N}>R_{1}-\epsilon \quad \text { and } \quad \frac{\left|\mathcal{G}^{(2)}\right|}{N}>R_{2}-\epsilon,
$$

where

$$
\begin{aligned}
& \mathcal{G}^{(1)}=\left\{i \in \mathcal{S}_{U}: I\left(S_{i} ; Y^{N} \mid S^{i-1}\right)>1-2^{-N^{\beta}}\right\}, \\
& \mathcal{G}^{(2)}=\left\{i \in \mathcal{S}_{V}: I\left(S_{i} ; Y^{N} \mid S^{i-1}\right)>1-2^{-N^{\beta}}\right\} .
\end{aligned}
$$

\section{Two-USER COMPound MAC}

We are now ready to described a polar coding scheme for the two-user compound MAC consisting of two channels $P_{Y}(y \mid x, w)$ and $P_{Z}(z \mid x, w)$. The channel is assumed to be known at the receiver but not at the transmitter. A rate pair $\left(R_{1}, R_{2}\right)$ is achievable if there exists a sequence of codes with rates approaching $\left(R_{1}, R_{2}\right)$ and vanishing error probability on both MACs. The capacity region is described by

$$
\bigcup_{p}\left(\mathscr{R}_{Y}(p) \cap \mathscr{R}_{Z}(p)\right),
$$

where $\mathscr{R}_{Y}(p)$ and $\mathscr{R}_{Z}(p)$ are as in (4). Recall that for the simple MAC, the time-sharing random variable $Q$ in (4) can be replaced by a convex hull operation on the union in (3). However, in the compound case, this substitution leads in general to a strictly smaller rate region.

\section{A. Uniform Independent Inputs}

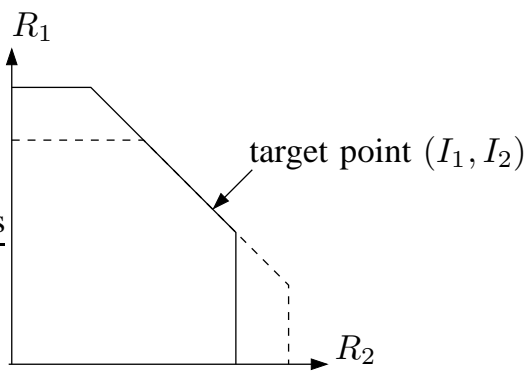

Fig. 3. Two MAC regions with equal sum-rate.

Assume $X$ and $W$ are uniform and independent, $Q=\emptyset$. The simplest nontrivial case is when the two pentagons in (4) intersect as in Figure 3, with equal sum-rate $I(X, W ; Y)=$ $I(X, W ; Z)$. Let $\left(I_{1}, I_{2}\right)$ be a rate point on the dominant face of this intersection. Let $U^{N}=X^{N} G_{N}$ and $V^{N}=W^{N} G_{N}$. By Proposition 11, there exists an $N$ and two monotone permutations $S^{2 N}$ and $T^{2 N}$ for which the mutual informations $I\left(S_{i} ; Y^{N} \mid S^{i-1}\right)$ and $I\left(T_{i} ; Z^{N} \mid T^{i-1}\right)$ are polarized, and the corresponding rate pairs in (5) are close to $\left(I_{1}, I_{2}\right)$. However, as in the point-to-point case, the two sets of mutual informations $\left\{I\left(S_{i} ; Y^{N} \mid S^{i-1}\right): i \in \mathcal{S}_{U}\right\}$ and $\left\{I\left(T_{i} ; Z^{N} \mid T^{i-1}\right): i \in\right.$ $\left.\mathcal{T}_{U}\right\}$ may be incompatible. One can similarly identify the type of index $i$ by comparing the mutual informations of the bit-channels $S_{j} \rightarrow Y^{N} S^{j-1}$ and $T_{k} \rightarrow Z^{N} T^{k-1}$, where $S_{j}=T_{k}=U_{i}$, and find the type II and type III incompatible index sets $\mathcal{A}_{\mathrm{II}}^{(1)}$ and $\mathcal{A}_{\mathrm{III}}^{(1)}$ for $U$, and $\mathcal{A}_{\mathrm{II}}^{(2)}$ and $\mathcal{A}_{\mathrm{III}}^{(2)}$ for $V$.

Now we apply the technique in Section II-A to align incompatible indices of both $U$ 's and $V$ 's. Here, as in the point-to-point case, care must be taken to combine the random variables in a way that guarantees successive decodability. This can be done by aligning only the U's or $V$ 's in any given recursion. Also, as before, only half of the incompatible indices of $U$ 's (or $V$ 's) are aligned in a single recursion. Aligning the two index sets alternately over $2 k$ recursions, both fractions of incompatible indices can be reduced to $1 / 2^{k}$ times their original values. 


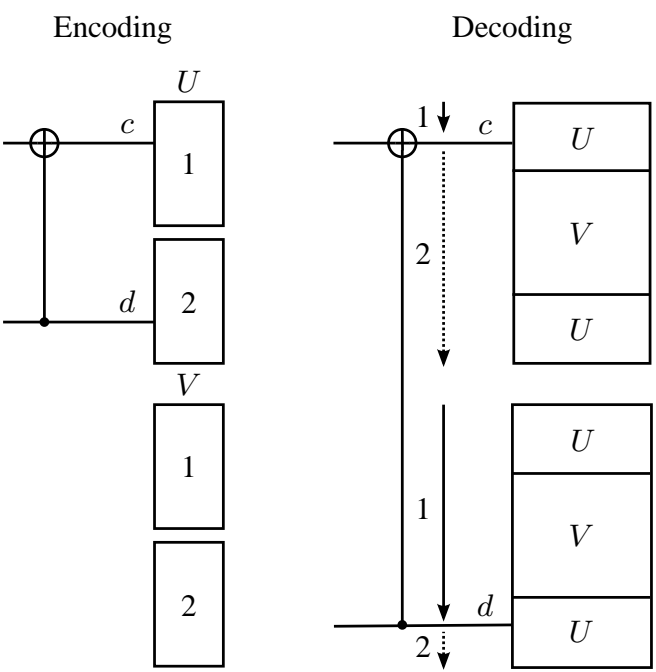

Fig. 4. First recursion.
Encoding
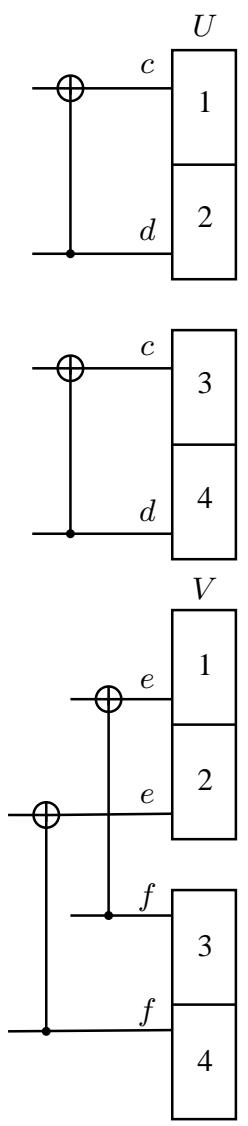

Decoding

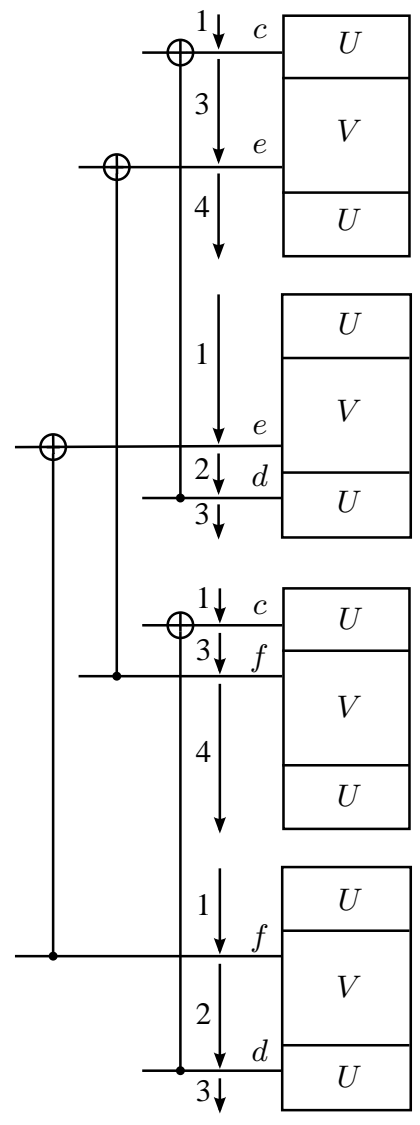

Fig. 5. Second recursion.
As an example, we show two recursions of alignment, where incompatible $U$ 's are aligned in the first recursion and incompatible $V$ 's are aligned in the second. Suppose $\mathcal{A}_{\mathrm{II}}^{(1)}=\{c\}, \mathcal{A}_{\mathrm{III}}^{(1)}=\{d\}, \mathcal{A}_{\mathrm{II}}^{(2)}=\{e\}, \mathcal{A}_{\mathrm{III}}^{(2)}=\{f\}$. In the first recursion (blocks 1 and 2), $U$ 's are aligned while $V$ 's are left uncombined (Figure 4). The decoding order for receiver 1 is shown on the right. After stacking the $U$ 's and $V$ 's according to the monotone permutation $S^{2 N}=\left(U^{i}, V^{N}, U_{i+1}^{N}\right)$, decoding can be proceeded in a similar fashion as in the pointto-point alignment (recall Figure 2). In the second recursion (Figure 5), a copy of the length- $2 N$ superblock is made (blocks 3 and 4) for both $U$ and $V$. The two superblocks of $V$ 's are aligned while the two superblocks of $U$ 's are left uncombined (as shown on the left). At the decoder 1, $U$ 's and $V$ 's from the same block are stacked according to the monotone permutation $S^{2 N}$. The uncombined indices in each block are decoded until reaching a combined index. Then the two combined indices are decoded. Since in each recursion, only incompatible indices for $U$ (or $V$ ) are combined in the right order, successive decodability is guaranteed as in the point-to-point case. More specifically for the running example, variables along an arrow with smaller number should be decoded before those with a bigger number, and variables along arrows with the same number can be decoded parallelly. The monotone permutation $S^{8 N}$ is defined by variables listed according to such a decoding order. The corresponding rate pair $\left(R_{1}^{s}, R_{2}^{s}\right)$ are defined as before

$$
\begin{aligned}
& R_{1}^{s}=\frac{1}{N} \sum_{i \in \mathcal{S}_{U}} I\left(S_{i} ; Y^{4 N} \mid S^{i-1}\right), \\
& R_{2}^{s}=\frac{1}{N} \sum_{i \in \mathcal{S}_{V}} I\left(S_{i} ; Y^{4 N} \mid S^{i-1}\right) .
\end{aligned}
$$

The decoding at the receiver 2 is performed according to the monotone permutation $T^{2 N}$ in the similar fashion. The resulting permutation $T^{8 N}$ and its rate pair $\left(R_{1}^{t}, R_{2}^{t}\right)$ can be defined similarly. Clearly, the fraction of incompatible indices for $U$ (and $V$ ) is halved in the first (second) recursion.

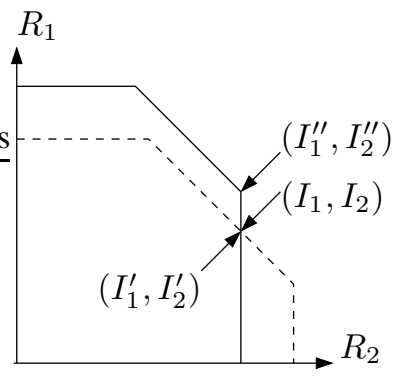

Fig. 6. Two MAC regions with unequal sum-rates.

To achieve a rate point $\left(I_{1}, I_{2}\right)$ in the general case where $I(X, W ; Y) \neq I(X, W ; Z)$ as in Figure 6 one can find two monotone permutations, which respectively approximate rate pairs $\left(I_{1}^{\prime}, I_{2}^{\prime}\right)$ on the dominant face of $P_{Y}(y \mid x, w)$ and $\left(I_{1}^{\prime \prime}, I_{2}^{\prime \prime}\right)$ on the dominant face of $P_{Z}(z \mid x, w)$, such that

$$
\begin{aligned}
& I_{1} \leq \min \left\{I_{1}^{\prime}, I_{1}^{\prime \prime}\right\}, \\
& I_{2} \leq \min \left\{I_{2}^{\prime}, I_{2}^{\prime \prime}\right\} .
\end{aligned}
$$

Then, applying the approach above achieves the target rate point. 


\section{B. Arbitrary Inputs}

Based on the polar coding scheme developed for uniform and independent $X$ and $W$, one can adapt the method in $[8$, Section III-D] to design a polar coding scheme for independent nonuniform $X$ and $W$. For correlated input distribution $\tilde{p}=p(q) p(x \mid q) p(w \mid q)$, there exist $\left(X^{\prime}, W^{\prime}, Q\right)$ mutually independent and functions $x\left(x^{\prime}, q\right)$ and $w\left(w^{\prime}, q\right)$ that induce the same distribution as $\tilde{p}$. Now consider a new MAC with inputs $X^{\prime}$ and $W^{\prime}$, vector output $(Y, Q)$, and conditional distribution $P^{\prime}\left(y, q \mid x^{\prime}, w^{\prime}\right)=p(q) P\left(y \mid x\left(x^{\prime}, q\right), w\left(w^{\prime}, q\right)\right)$, where $Q$ is the common randomness shared at the senders and the receiver. Then the achievable rate region for the new MAC is the set of rate pairs $\left(R_{1}, R_{2}\right)$ such that

$$
\begin{aligned}
R_{1} & \leq I\left(X^{\prime} ; Y, Q, W^{\prime}\right)=I(X ; Y, W \mid Q), \\
R_{2} \leq I\left(W^{\prime} ; Y, Q, X^{\prime}\right) & =I(W ; Y, X \mid Q), \\
R_{1}+R_{2} \leq I\left(X^{\prime}, W^{\prime} ; Y, Q\right) & =I(X, W ; Y \mid Q)
\end{aligned}
$$

for distribution $p^{\prime}=p(q) p\left(w^{\prime}\right) p\left(x^{\prime}\right) p\left(x \mid x^{\prime}, q\right) p\left(w \mid w^{\prime}, q\right)$ $P\left(y \mid x\left(x^{\prime}, q\right), w\left(w^{\prime}, q\right)\right)$, where $p\left(x \mid x^{\prime}, q\right)$ and $p\left(w \mid w^{\prime}, q\right)$ are $\{0,1\}$-valued according to $x\left(x^{\prime}, q\right)$ and $w\left(w^{\prime}, q\right)$. This rate region is identical to $\mathscr{R}_{Y}(p)$ as $p^{\prime} \equiv p$. Similarly the rate region $\mathscr{R}_{Y}(p) \cap \mathscr{R}_{Z}(p)$ can be described by considering the compound MAC with inputs $X^{\prime}$ and $W^{\prime}$, vector output $(Y, Z, Q)$, and conditional distribution $P^{\prime}\left(y, z, q \mid x^{\prime}, w^{\prime}\right)=$ $p(q) P_{Y}\left(y \mid x\left(x^{\prime}, q\right), w\left(w^{\prime}, q\right)\right) P_{Z}\left(z \mid x\left(x^{\prime}, q\right), w\left(w^{\prime}, q\right)\right)$. One can apply the method designed for independent input to achieve arbitrary point in the rate region of the new compound MAC. To complete the proof, one just need to show the existence of a good common random sequence $q^{n}$, which is shared at the senders and the receiver before the transmission. This is guaranteed since the average probability of error over all possible choices of $q^{n}$ is small.

\section{Main Result}

Theorem 1. For every $\epsilon>0, \beta<1 / 2$, and rate pair $\left(I_{1}, I_{2}\right)$ in the rate region $\mathscr{R}_{Y}(p) \cap \mathscr{R}_{Z}(p)$, there exist $N, M=2^{k} N$, and two monotone permutation $S^{2 M}$ and $T^{2 M}$ with associated rate pairs $\left(R_{1}^{s}, R_{2}^{s}\right)$ and $\left(R_{1}^{t}, R_{2}^{t}\right)$ such that for $j=1,2$,

$$
\left|\min \left\{R_{j}^{s}, R_{j}^{t}\right\}-I_{j}\right|<\epsilon,
$$

$$
\frac{\left|\mathcal{G}_{Y}^{(j)} \cap \mathcal{G}_{Z}^{(j)}\right|}{M}>\min \left\{R_{j}^{s}, R_{j}^{t}\right\}-\epsilon,
$$

where

$$
\begin{array}{r}
\mathcal{G}_{Y}^{(1)}=\left\{i \in \mathcal{S}_{U}: I\left(S_{i} ; Y^{M} \mid S^{i-1}\right)>1-2^{-N^{\beta}}\right\}, \\
\mathcal{G}_{Z}^{(1)}=\left\{i \in \mathcal{T}_{U}: I\left(T_{i} ; Z^{M} \mid T^{i-1}\right)>1-2^{-N^{\beta}}\right\}, \\
\mathcal{G}_{Y}^{(2)}, \mathcal{G}_{Z}^{(2)} \text { are defined similarly by replacing } U \text { by } V .
\end{array}
$$

The above theorem implies that arbitrary point in the capacity region of the two-user compound MAC is achievable with the proposed polar coding scheme. In the two-user strong interference channel, that is $I(X ; Y, W) \leq I(X ; Z, W)$ and $I(W ; Z, X) \leq I(W ; Y, X)$ for all $p(x) p(w)$, decoding both messages at each receiver is optimal and the two-user compound MAC region coincides with the capacity region of the interference channel. Therefore, the same technique applies to the two-user strong interference channels.

\section{INTERFERENCE NETWORKS}

Now we generalize the result to $K$-sender $L$-receiver interference networks with input alphabets $\mathcal{X}_{1}, \ldots, \mathcal{X}_{K}$, and output alphabets $\mathcal{Y}_{1}, \ldots, \mathcal{Y}_{L}$, and conditional distribution $P\left(y^{L} \mid x^{K}\right)$ as depicted in Figure 7 Each sender $j \in[1: K]$ com-

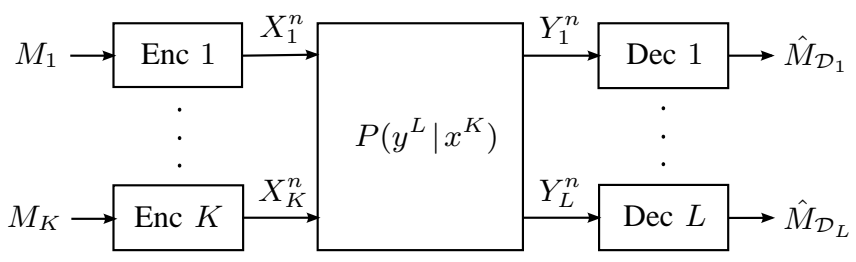

Fig. 7. $K$-sender $L$-receiver interference networks.

municates an independent message $M_{j}$ at rate $R_{j}$ and each receiver $l \in[1: L]$ wishes to recover a subset $\mathcal{D}_{l} \subseteq[1: K]$ of the messages. The optimal rate region when the encoding is restricted to random coding ensembles with superposition coding and time sharing [9] is the union over $\left\{\left(\mathcal{A}_{1}, \ldots, \mathcal{A}_{L}\right)\right.$ : $\left.\mathcal{A}_{l} \supseteq \mathcal{D}_{l}, l \in[1: L]\right\}$ of the region

$$
\bigcap_{l \in[1: L]} \mathscr{R}_{\mathcal{A}_{l}}(p)
$$

where the input distribution is of the form $p=p(q)$ $\left(\prod_{j=1}^{K} p\left(x_{j} \mid q\right)\right) P\left(y^{L} \mid x^{K}\right)$ and $\mathscr{R}_{\mathcal{A}_{l}}(p)$ is the set of rate tuples $\left(R_{j}: j \in \mathcal{A}_{l}\right)$ such that

$$
R(\mathcal{J}) \leq I\left(X_{\mathcal{J}} ; Y_{l}, X_{\mathcal{A}_{l} \backslash \mathcal{J}} \mid Q\right)
$$

for all $\mathcal{J} \subseteq \mathcal{A}_{l}$. Here we introduce notation

$$
R(\mathcal{J}):=\sum_{j \in \mathcal{J}} R_{j}
$$

and

$$
X_{\mathcal{J}}:=\left(X_{j}: j \in \mathcal{J}\right)
$$

for an index set $\mathcal{J}$. It is clear from (7) that this rate region is also a compound MAC region.

To apply the proposed polar coding scheme to the interference networks, one needs to (i) generalize Arıkan's polar splitting result to $K$-user MAC and (ii) align more than two incompatible polarization processes, each of which involves codes from $K$ users. We prove (i) in Section IV-A and discuss (ii) in Section IV-B We show two important applications in Sections IV-C and IV-D

\section{A. 'Polar Splitting' for K-user MAC}

Consider a $K$-user MAC, where transmitter $j, j \in[1: K]$, wishes to communicate a message $M_{j}$ reliably to the receiver by sending a codeword $X_{j}^{N}\left(M_{j}\right)=\left(X_{j 1}, X_{j 2}, \ldots, X_{j N}\right)$ over the memoryless channel $P\left(y \mid x^{K}\right)$. The receiver wishes 
to recover all the messages $M_{[1: K]}$. The capacity region of the $K$-user MAC is described by

$$
\bigcup_{p} \mathscr{R}_{[1: K]}(p),
$$

where the union is over all distributions of the form $p=$ $p(q)\left(\prod_{i=1}^{K} p\left(x_{i} \mid q\right)\right) P\left(y \mid x^{K}\right)$, and $\mathscr{R}_{[1: K]}(p)$ is defined as in (8).

Let $U_{j}^{N}=X_{j}^{N} G_{N}$ for $j \in[1: K]$. Similar to the two-user MAC case, we have the chain rule of the form

$$
\sum_{i=1}^{K N} I\left(S_{i} ; Y^{N} \mid S^{i-1}\right),
$$

where $S^{K N}$ is a monotone permutation of $\left(U_{1}^{N}, \ldots, U_{K}^{N}\right)$, i.e., elements of $U_{j}^{N}$ appear in increasing order in $S^{K N}$ for all $j \in$ $[1: K]$. Let $\mathcal{S}_{j}$ denote the index set $\left\{i: S_{i}=U_{j k}\right.$ for some $\left.k\right\}$. Define the associated rate tuple $\left(R_{1}, \ldots, R_{K}\right)$ of the monotone permutation as

$$
R_{j}=\frac{1}{N} \sum_{i \in \mathcal{S}_{j}} I\left(S_{i} ; Y^{N} \mid S^{i-1}\right)
$$

for $j \in[1: K]$. We now generalize Arıkan's polar-splitting result to $K$ users.

Proposition 2. For every $\epsilon>0, \beta<1 / 2$, and rate tuple $\left(I_{1}, \ldots, I_{K}\right)$ on the dominant face of $\mathscr{R}_{[1: K]}(p)$, there exists an $N$ and a monotone permutation $S^{K N}$ such that for all $j \in[1: K]$,

(i)

(ii)

$$
\left|R_{j}-I_{j}\right| \leq \epsilon,
$$

$$
\frac{\left|\mathcal{G}^{(j)}\right|}{N}>R_{j}-\epsilon,
$$

where

$$
\mathcal{G}^{(j)}=\left\{i \in \mathcal{S}_{j}: I\left(S_{i} ; Y^{N} \mid S^{i-1}\right)>1-2^{-N^{\beta}}\right\} .
$$

Proof: We prove statement (i) by induction. The case $K=2$ holds by Proposition 1 Suppose the statement holds up to $K-1$. We prove the statement for $K$.

Assume without loss of generality that we start by decoding $U_{1}^{i_{0}}$ for some $i_{0} \in[1: N]$. We specify $i_{0}$ by the following procedure. Let $i$ increase from 0 to $N$ and consider the quantities

$$
\frac{1}{N} I\left(U_{\mathcal{J}}^{N} ; Y^{N}, U_{1}^{i}\right)
$$

for each $\mathcal{J} \subseteq[2: N]$. Some observations follow:

1) As $i$ increases, each mutual information term increases by at most $1 / N$ in each step, since the increment is $I\left(U_{1 i} ; U_{\mathcal{J}}^{N} \mid Y^{N}, U_{1}^{i-1}\right) / N \leq 1 / N$.

2) There exists an $i$ such that for at least one $\mathcal{J} \subseteq[2: K]$, the following is violated

$$
\frac{1}{N} I\left(U_{\mathcal{J}}^{N} ; Y^{N}, U_{1}^{i}\right)<I(\mathcal{J}) .
$$

To see 2), set $U_{1}^{i}=\emptyset$ and $U_{1}^{i}=U_{1}^{N}$ respectively. We have

$$
\begin{aligned}
\frac{1}{N} I\left(U_{\mathcal{J}}^{N} ; Y^{N}\right) & \leq I(\mathcal{J}) \quad \text { for } \mathcal{J} \subset[2: K], \\
\frac{1}{N} I\left(U_{[2: K]}^{N} ; Y^{N}\right) & \leq I([2: K]) \leq I\left(U_{[2: K]}^{N} ; Y^{N}, U_{1}^{N}\right) .
\end{aligned}
$$

As $i$ increases, the mutual information terms in (9) increase steadily. Therefore, there exists an $i$ such that (10) is violated for some $\mathcal{J} \subseteq[2: K]$. Take the smallest such $i$ as $i_{0}$.

Suppose at $i=i_{0}$, the inequality in (10) is violated at $\mathcal{J}_{0}$ for the first time. As the increment on the left-hand-side of (10) is bounded by $1 / N$, we roughly have

$$
\frac{1}{N} I\left(U_{\mathcal{J}_{0}}^{N} ; Y^{N}, U_{1}^{i_{0}}\right)=I\left(\mathcal{J}_{0}\right) .
$$

This divides the $K$-dimensional rate-approximation into two subproblems of smaller dimensions.

Problem 1: For $\mathcal{J} \subseteq \mathcal{J}_{0}$, we have

$$
\frac{1}{N} I\left(U_{\mathcal{J}}^{N} ; Y^{N}, U_{1}^{i_{0}}\right)<I(\mathcal{J}) \text { for all } \mathcal{J} \subset \mathcal{J}_{0}
$$

and

$$
\frac{1}{N} I\left(U_{\mathcal{J}_{0}}^{N} ; Y^{N}, U_{1}^{i_{0}}\right)=I\left(\mathcal{J}_{0}\right) .
$$

This is a rate-approximation problem for the rate tuple $\left(I_{j}: j \in \mathcal{J}_{0}\right)$ on the dominant face of a $\left|\mathcal{J}_{0}\right|$-user MAC with output $\left(Y^{N}, U_{1}^{i_{0}}\right)$.

Problem 2: For all $\mathcal{J} \supseteq \mathcal{J}_{0}$, we subtract (11) from (10). Let $\mathcal{T}=\mathcal{J} \backslash \mathcal{J}_{0}, \mathcal{T}_{0}=[2: K] \backslash \mathcal{J}_{0}$, and $I_{1}^{\prime}=I_{1}-\frac{1}{N} I\left(U_{1}^{i_{0}} ; Y^{N}\right)$. This yields

$$
\begin{aligned}
\frac{1}{N} I\left(U_{\mathcal{T}}^{N} ; Y^{N}, U_{\mathcal{J}_{0}}^{N}, U_{1}^{i_{0}}\right) & \leq I(\mathcal{T}), \\
\frac{1}{N} I\left(U_{\mathcal{T}}^{N}, U_{1, i_{0}+1}^{N} ; Y^{N}, U_{\mathcal{J}_{0}}^{N}, U_{1}^{i_{0}}\right) & \leq I(\mathcal{T})+I_{1}^{\prime}, \\
\frac{1}{N} I\left(U_{\mathcal{T}_{0}}^{N}, U_{1, i_{0}+1}^{N} ; Y^{N}, U_{\mathcal{J}_{0}}^{N}, U_{1}^{i_{0}}\right) & =I\left(\mathcal{T}_{0}\right)+I_{1}^{\prime} .
\end{aligned}
$$

This is a rate-approximation problem for the rate tuple $\left(I_{1}^{\prime},\left(I_{j}: j \in \mathcal{T}_{0}\right)\right)$ on the dominant face of a $\left(K-\left|\mathcal{J}_{0}\right|\right)$-user MAC with output $\left(Y^{N}, U_{1}^{i_{0}}, U_{\mathcal{J}_{0}}^{N}\right)$.

Note that $1 \leq\left|\mathcal{J}_{0}\right| \leq K-1$. Thus both problems are reduced to a smaller dimension. The final path is obtained by cascading $1^{i_{0}}, b^{\left|\mathcal{J}_{0}\right| N}$ (the solution from problem 1), and $b^{K N-\left|\mathcal{J}_{0}\right| N-i_{0}}$ (the solution from problem 2).

The polarization result (ii) is obtained by standard path scaling as in [5]. This concludes the proof.

\section{B. Aligning polarized indices for $K$ users}

Suppose we have two monotone permutations for two $K$ user MACs. To align the incompatible indices for all users, one can continue the method in Section $\amalg-A$ and sequentially align the incompatible indices for each $U_{j}^{N}, j \in[1: K]$. After alternately aligning $K$ index sets over $K m$ recursions, the fraction of the incompatible indices for each user is reduced to $1 / 2^{m}$ times the original fraction. The method for aligning $L$ monotone permutations can be done by recursively aligning two permutations as in [6].

\section{Han-Kobayashi Inner bound}

As an important special case, we show how the scheme above can be used to achieve the Han-Kobayashi inner bound, the best known inner bound for general two-user interference channels $P\left(y_{1}, y_{2} \mid x_{1}, x_{2}\right)$.

The Han-Kobayashi coding scheme is illustrated in Figure 8 Message $M_{1}$ is split into two independent parts $\left(L_{1}, L_{2}\right)$ and message $M_{2}$ is split into two independent parts $\left(L_{3}, L_{4}\right)$. Message $L_{j}, j \in[1: 4]$, is carried by codeword $V_{j}^{N}\left(L_{j}\right)$. 


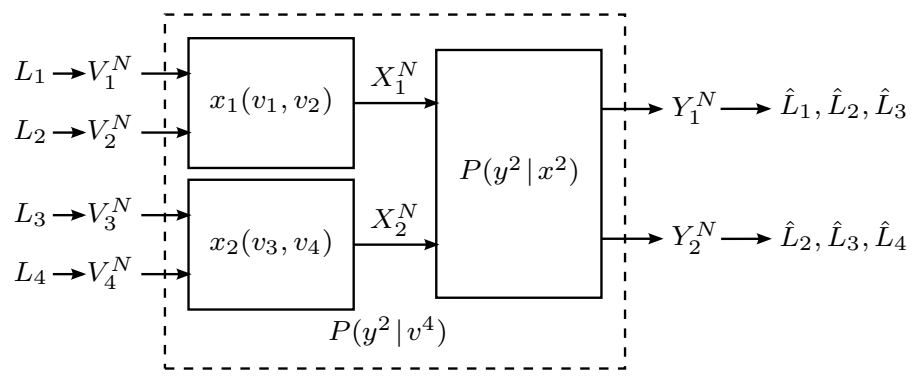

Fig. 8. Han-Kobayashi coding scheme.

Then the channel inputs $X_{1}^{N}$ and $X_{2}^{N}$ are formed using two symbol-by-symbol mappings $x_{1}\left(v_{1}, v_{2}\right)$ and $x_{2}\left(v_{3}, v_{4}\right)$. Receiver 1 uniquely decodes $\left(\hat{L}_{1}, \hat{L}_{2}, \hat{L}_{3}\right)$ upon receiving $Y_{1}^{N}$, while receiver 2 uniquely decodes $\left(\hat{L}_{2}, \hat{L}_{3}, \hat{L}_{4}\right)$ upon receiving $Y_{2}^{N}$. The achievable rate region of the Han-Kobayashi coding scheme is given by

$$
\bigcup_{p} \operatorname{Proj}_{4 \rightarrow 2}\left(\mathscr{R}_{1}(p) \cap \mathscr{R}_{2}(p)\right) .
$$

Here the input distribution is of the form $p=p(q)$ $\left(\prod_{j=1}^{4} p\left(v_{j} \mid q\right)\right) p\left(x_{1} \mid v_{1}, v_{2}, q\right) p\left(x_{2} \mid v_{3}, v_{4}, q\right) P\left(y_{1}, y_{2} \mid x_{1}, x_{2}\right)$, where $p\left(x_{1} \mid v_{1}, v_{2}, q\right)$ and $p\left(x_{2} \mid v_{3}, v_{4}, q\right)$ are $\{0,1\}$-valued according to functions $x_{1}\left(v_{1}, v_{2}, q\right)$ and $x_{2}\left(v_{3}, v_{4}, q\right)$. The rate region $\mathscr{R}_{1}(p)$ is the set of rate triples $\left(R_{1}^{\prime}, R_{2}^{\prime}, R_{3}^{\prime}\right)$ such that

$$
R_{\mathcal{J}}^{\prime} \leq I\left(V(\mathcal{J}) ; Y_{1}^{N}, V([1: 3] \backslash \mathcal{J}) \mid Q\right)
$$

for all $\mathcal{J} \subseteq[1: 3]$. The rate region $\mathscr{R}_{2}(p)$ is the set of rate triples $\left(R_{2}^{\prime}, R_{3}^{\prime}, R_{4}^{\prime}\right)$ such that

$$
R_{\mathcal{J}}^{\prime} \leq I\left(V(\mathcal{J}) ; Y_{2}^{N}, V([2: 4] \backslash \mathcal{J}) \mid Q\right)
$$

for all $\mathcal{J} \subseteq[2: 4]$. The operator $\operatorname{Proj}_{4 \rightarrow 2}$ is to apply the Fourier-Motzkin elimination from the 4-dimensional space $\left(R_{1}^{\prime}, R_{2}^{\prime}, R_{3}^{\prime}, R_{4}^{\prime}\right)$ to the 2 -dimensional space $\left(R_{1}, R_{2}\right)$ by setting $R_{1}=R_{1}^{\prime}+R_{2}^{\prime}$ and $R_{2}=R_{3}^{\prime}+R_{4}^{\prime}$.

It is clear from the Han-Kobayashi coding scheme that for each pair of functions $x_{1}\left(v_{1}, v_{2}\right)$ and $x_{2}\left(v_{3}, v_{4}\right)$, the message splitting transforms the the original two-user interference channel into a four-sender two-receiver interference networks

$$
P\left(y^{2} \mid v^{4}\right)=P\left(y_{1}, y_{2} \mid x_{1}\left(v_{1}, v_{2}\right), x_{2}\left(v_{3}, v_{4}\right)\right),
$$

where sender $j \in\{1,2,3,4\}$ communicates an independent message $L_{j}$ at rate $R_{j}^{\prime}$, receiver 1 recovers the subset $\mathcal{D}_{1}=$ $\{1,2,3\}$ of the four messages, and receiver 2 recovers the sucset $\mathcal{D}_{2}=\{2,3,4\}$ of the four messages.

Note from expression (12) that the auxiliary rate region $\left(R_{1}^{\prime}, R_{2}^{\prime}, R_{3}^{\prime}, R_{4}^{\prime}\right)$ is the intersection of two 3-dimensional MAC regions, two dimensions of which are in common. Therefore, one just needs to find two monotone permutations that achieves any target point in the two MACs respectively and sequentially align the two codes shared in common using the method in Section III-A.

\section{Superposition Coding for Broadcast Channels}

The method for interference networks also implies the achievability of the superposition coding inner bound for general broadcast channels. As the simplest example, consider a two-user broadcast channel $P\left(y_{1}, y_{2} \mid x\right)$, where the sender wishes to communicate message $M_{1}$ to receiver 1 and message $M_{2}$ to receiver 2 .

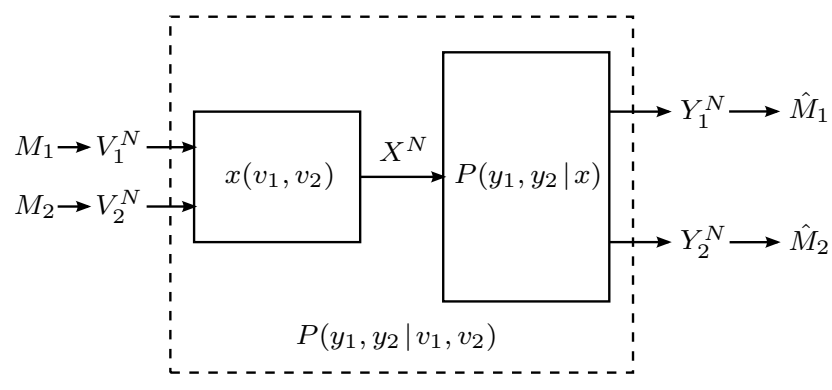

Fig. 9. Cover's homogeneous superposition coding.

Cover's homogeneous superposition coding [10] is illustrated in Figure 9 . Two auxiliary sequences of codewords $V_{1}^{N}\left(M_{1}\right)$ and $V_{2}^{N}\left(M_{2}\right)$ are generated according to independent distribution $p\left(v_{1}\right) p\left(v_{2}\right)$. Then the channel input $X^{N}$ is formed through the symbol-by-symbol mapping $x\left(v_{1}, v_{2}\right)$. This transforms the broadcast channel into a two-sender tworeceiver interference networks

$$
P\left(y_{1}, y_{2} \mid v_{1}, v_{2}\right)=P\left(y_{1}, y_{2} \mid x\left(v_{1}, v_{2}\right)\right),
$$

where sender $j \in\{1,2\}$ communicates an independent message $M_{j}$ and receiver $j \in\{1,2\}$ recovers a subset $\mathcal{D}_{j}=\{j\}$ of the messages. Clearly, this is another special case of the interference networks.

It is worth mention that compared to Bergmans's heterogeneous superposition coding [11], where the codeword $X^{N}\left(M_{1}, M_{2}\right)$ is generated conditioned on the codeword $V^{N}\left(M_{1}\right)$ according to distribution $p(v) p(x \mid v)$, Cover's homogeneous superposition coding achieves in general a strictly larger rate region in the two-user broadcast channels under optimal decoding [12]. The rate region achievable by Cover's superposition encoding and optimal decoding is [12]

$$
\bigcup_{p} \bigcup_{i=1}^{4}\left(\mathscr{R}_{1 i}(p) \cap \mathscr{R}_{2 i}(p)\right),
$$

where the distribution is of the form $p=p\left(v_{1}\right) p\left(v_{2}\right)$ $p\left(x \mid v_{1}, v_{2}\right) P\left(y_{1}, y_{2} \mid x\right)$ with $\{0,1\}$-valued $p\left(x \mid v_{1}, v_{2}\right)$ and $\mathscr{R}_{1 i}(p) \cap \mathscr{R}_{2 i}(p)$ corresponds to the rate region when the decoders are required to uniquely recover the following message sets

$$
\begin{aligned}
& i=1: \mathcal{A}_{1}=\{1\}, \mathcal{A}_{2}=\{2\} ; \\
& i=2: \mathcal{A}_{1}=\{1,2\}, \mathcal{A}_{2}=\{2\} ; \\
& i=3: \mathcal{A}_{1}=\{1\}, \mathcal{A}_{2}=\{1,2\} ; \\
& i=4: \mathcal{A}_{1}=\{1,2\}, \mathcal{A}_{2}=\{1,2\} .
\end{aligned}
$$

For example, $\mathscr{R}_{13}(p) \cap \mathscr{R}_{23}(p)$ is the set of $\left(R_{1}, R_{2}\right)$ such that

$$
R_{1}<I\left(V_{1} ; Y_{1}\right),
$$




$$
\begin{aligned}
R_{1} & <I\left(V_{1} ; Y_{2}, V_{2}\right), \\
R_{2} & <I\left(V_{2} ; Y_{2}, V_{1}\right), \\
R_{1}+R_{2} & <I\left(V_{1}, V_{2} ; Y_{2}\right) .
\end{aligned}
$$

To achieve arbitrary point here, one can first find a good pointin-point code for $\mathscr{R}_{13}(p)$ and a monotone permutation for the MAC region $\mathscr{R}_{23}(p)$. Then apply method in $\amalg-\mathrm{A}$ to align the incompatible indices in the code for $V_{1}^{N}$. This achieves any point in the rate region $\mathscr{R}_{13}(p) \cap \mathscr{R}_{23}(p)$. Similarly for each decoding set, one can design a corresponding polar coding scheme based on the method above. Therefore, the proposed polar coding scheme achieves the optimal rate region given Cover's superposition encoding. The generalization to $L$-user broadcast channels can be done similarly.

As a side remark, the independence between $V_{1}$ and $V_{2}$ in Cover's superposition coding is important for transforming the broadcast channel into a two-user interference channel. For general correlated $\left(V_{1}, V_{2}\right) \sim p\left(v_{1}, v_{2}\right)$ as in Marton coding for broadcast channels, one needs different techniques. A method for Marton coding as well as an alternative polar coding scheme for Bergmans's superposition coding can be found in [13].

\section{Discussion}

We have shown a polar coding method for the general interference networks that achieves the optimal rate region when the encoding is restricted to random coding ensembles with superposition coding and time sharing [9]. As special cases, the method achieves the capacity region of the compound MAC, the Han-Kobayashi inner bound for twouser interference channels, and the superposition coding inner bound for broadcast channels.

One drawback of the current method is the long blocklength needed for large scale networks. When there are $L$ receivers in the networks, one needs to do $L-1$ alignments to resolve the incompatible indices in $L$ permutations, which makes the blocklength scale with the network size.

One crucial component in the current method is Arıkan's 'polar splitting' for MAC. It would be interesting to compare it to regular rate splitting for MAC as in [3]. Both schemes achieve optimal performance in MAC. However, for interference channels, the former, together with the alignment method, achieves the best known rate region while the latter is strictly suboptimal information theoretically [4].
In a parallel study [4], a successive decoding based random coding scheme is presented, which also achieves HanKobayashi inner bound. Some similarities and connections can be found in the way the two schemes resolve the incompatibility of the two MACs.

\section{ACKNOWLEDGEMENT}

L. Wang would like to thank Young-Han Kim for suggesting the problem and the guidance throughout. Her work is supported by the 2013 Qualcomm Innovation Fellowship, the SAMSUNG Global Research Outreach program, and the National Science Foundation under grant CCF-1116139. E. Şaşoğlu's work is supported by the Swiss National Science Foundation under grant PBELP2_137726.

\section{REFERENCES}

[1] T. S. Han and K. Kobayashi, "A new achievable rate region for the interference channel," IEEE Trans. Inf. Theory, vol. 27, no. 1, pp. 4960, 1981.

[2] E. Şaşoğlu, E. Telatar, and E. Yeh, "Polar codes for the two-user multiple-access channel," Information Theory, IEEE Transactions on, vol. 59, no. 10, pp. 6583-6592, 2013.

[3] A. J. Grant, B. Rimoldi, R. L. Urbanke, and P. A. Whiting, "Ratesplitting multiple access for discrete memoryless channels," IEEE Trans. Inf. Theory, vol. 47, no. 3, pp. 873-890, 2001.

[4] L. Wang, E. Şaşoğlu, and Y.-H. Kim, "Sliding-window rate-splitting for interference networks," 2014, submitted to ISIT 2014.

[5] E. Arıkan, "Polar coding for the slepian-wolf problem based on monotone chain rules," in Proc. IEEE Int. Symp. Inf. Theory, Cambridge, MA, 2012, pp. 566-570.

[6] S. H. Hassani and R. L. Urbanke, "Universal polar codes," 2013. [Online]. Available: http://arxiv.org/abs/1307.7223

[7] S. Hassani, S. Korada, and R. L. Urbanke, "The compound capacity of polar codes," in Proc. 47th Ann. Allerton Conf. Comm. Control Comput., Monticello, IL, Sep. 2009, pp. 16-21.

[8] E. Şaşoğlu, E. Telatar, and E. Arikan, "Polarization for arbitrary discrete memoryless channels," 2009. [Online]. Available: http://arxiv.org/abs/0908.0302

[9] B. Bandemer, A. El Gamal, and Y.-H. Kim, "Optimal achievable rates for interference networks with random codes," 2012, preprint available at http://arxiv.org/abs/1210.4596/.

[10] T. M. Cover, "Broadcast channels," IEEE Trans. Inf. Theory, vol. 18, no. 1, pp. 2-14, Jan. 1972.

[11] P. P. Bergmans, "Random coding theorem for broadcast channels with degraded components," IEEE Trans. Inf. Theory, vol. 19, no. 2, pp. 197207, 1973.

[12] L. Wang, E. Şaşoğlu, B. Bandemer, and Y.-H. Kim, "A comparison of superposition coding schemes," in Proc. IEEE Int. Symp. Inf. Theory, Istanbul, Turkey, Jul. 2013.

[13] M. Mondelli, S. H. Hassani, I. Sason, and R. L. Urbanke, "Achieving the superposition and binning regions for broadcast channels using polar codes,” 2014. [Online]. Available: http://arxiv.org/abs/1401.6060 